\title{
Factors governing selectivity of dopamine receptor binding compounds for D2R and D3R subtypes
}

\author{
Hamed S. Hayatshahi ${ }^{1}$, Robert R. Luedtke ${ }^{2}$, Michelle Taylor ${ }^{2}$, Peng-Jen Chen ${ }^{3}$, Benjamin E. Blass ${ }^{3}$, Jin Liu ${ }^{1, *}$
}

${ }^{1}$ Department of Pharmaceutical Sciences, University of North Texas System College of Pharmacy, University of North Texas Health Science Center, 3500 Camp Bowie Blvd, Fort Worth, TX 76107, USA

${ }^{2}$ Department of Pharmacology and Neuroscience, University of North Texas Health Science Center, 3500 Camp Bowie Blvd, Fort Worth, TX 76107, USA

${ }^{3}$ Moulder Center for Drug Discovery Research, Department of Pharmaceutical Sciences, Temple University School of Pharmacy, 3307 North Broad Street, Philadelphia, PA 19140, USA

* To whom correspondence should be addressed: jin.liu@unthsc.edu

\section{Supporting Information}

\section{Details of synthesis of compounds 20 through 220:}

Scheme S1 shows the methods used for the synthesis of compounds 2o-22o. Reagents were purchased from Fisher Scientific, VWR International, Sigma Aldrich, and Combi-Blocks, Inc. Chromatographic purification of compounds (normal phase and reverse phase) were carried out on a Teledyne Isco Combiflash RF system. ${ }^{1} \mathrm{H}-\mathrm{NMR}$ spectra were obtained on a Bruker 300-MHz NMR. Chemical shift values ( $\delta$ values) were reported in ppm relative to TMS. For multiplicity, $\mathrm{s}=$ singlet, $\mathrm{d}=$ doublet, $\mathrm{t}=$ triplet, $\mathrm{m}=$ multiplet. Purity (\%) and mass spectral data were determined with a Waters Agilent 1200 HPLC/MS (Zorbax SB-C18, 2.1 x $30 \mathrm{~mm}, 3.5 \mu \mathrm{m}, 100 \%$ water $/ 0.1 \%$ formic acid to $100 \%$ acetonitrile $/ 0.1 \%$ formic acid over 4.0 minutes, $1.0 \mathrm{~mL} / \mathrm{min}$.) with a diode array detector from $210-400 \mathrm{~nm}$ and Agilent 6130 quadrupole MS. All compounds were purified to $95 \%$ purity or greater as determined by HPLC/MS and ${ }^{1} \mathrm{H}-\mathrm{NMR}$.

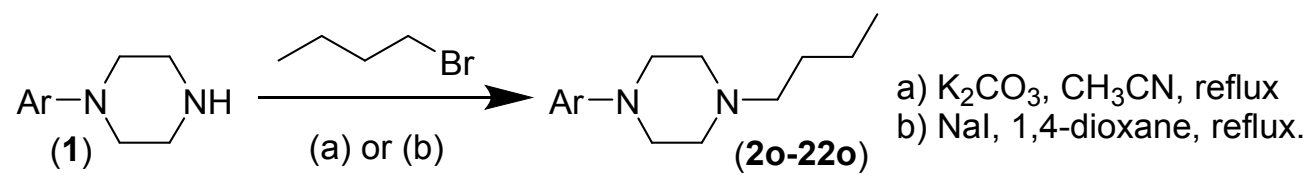

Scheme S1: Synthesis of substituted phenylpiperazines (20-22o)

Method (A): A solution of an aryl piperazine ((1) $1.095 \mathrm{mmoles})$, bromobutane $(0.07 \mathrm{ml}, 0.365 \mathrm{mmol})$, and potassium carbonate $(0.2 \mathrm{~g}$, $1.46 \mathrm{mmol}, 4.0 \mathrm{eq}$ ) in $6 \mathrm{~mL}$ of acetonitrile was heated to reflux. After 16 hours, the reaction was cooled, filtered to remove solid materials, and stripped of solvent. The remaining material was purified by normal phase chromatography $(10 \%$ methanol $/ 0.1 \%$ ammonium hydroxide in dichloromethane) or reverse phase chromatography ( $5 \%$ acetonitrile $/ 0.1 \%$ formic acid in water) as necessary to provide the desired product.

Method (B): A solution of 1-bromobutane $(0.1 \mathrm{ml}, 0.932 \mathrm{mmol})$, an aryl piperazine $((\mathbf{1}), 0.932 \mathrm{mmol})$ and sodium iodide $(0.83 \mathrm{~g}, 5.54$ $\mathrm{mmol}$ ) in anhydrous 1,4-dioxane was refluxed at $101{ }^{\circ} \mathrm{C}$ for 24 hours. The reaction was then cooled to $23^{\circ} \mathrm{C}$, filtered to remove solid precipitates, and stripped of solvents. The remaining material was purified by normal phase chromatography $(10 \%$ methanol/0.1\% 
ammonium hydroxide in dichloromethane) or reverse phase chromatography ( $5 \%$ acetonitrile $/ 0.1 \%$ formic acid in water) as necessary to provide the desired product.

Example 2o: 3-(4-butylpiperazin-1-yl)benzonitrile: ${ }^{20}$ The title compound was preapred according using method A, 78\% yield, ${ }^{1} H N M R$ $(400 \mathrm{MHz}, \mathrm{CDCl}) \delta 7.34-7.28(\mathrm{~m}, \mathrm{lH}), 7.12-7.08(\mathrm{~m}, 3 \mathrm{H}), 3.25(\mathrm{t}, \mathrm{J}=5 \mathrm{~Hz}, 4 \mathrm{H}), 2.61(\mathrm{t}, \mathrm{J}=5.12 \mathrm{~Hz}, 4 \mathrm{H}), 2.41(t, J=9.76 \mathrm{~Hz}, 2 \mathrm{H})$, 1.57-1.49 (m, $2 H), 1.41-1.32(m, 2 H), 0.95(t, J=7.32,3 H)$; $M S\left(L C / M S, M+H^{+}\right): 245$, HPLC purity: $98 \%$.

Example 3o: 2-(4-butylpiperazin-1-yl)benzonitrile: The title compound was preapred according using method A, 64\% yield. ${ }^{1} H N M R(400$

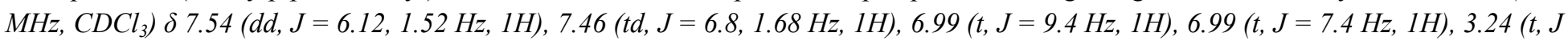
$=4.76 \mathrm{~Hz}, 4 \mathrm{H}), 2.66(t, J=4.88 \mathrm{~Hz}, 4 \mathrm{H}), 2.41(t, J=7.64 \mathrm{~Hz}, 2 \mathrm{H}), 1.55-1.47(\mathrm{~m}, 2 \mathrm{H}), 1.35-1.30(\mathrm{~m}, 2 \mathrm{H}), 0.93(t, J=7.32 \mathrm{~Hz}, 3 \mathrm{H}) ; \mathrm{MS}$ $\left(L C / M S, M+H^{+}\right): 245$, HPLC purity: $98 \%$.

Example 4o: 4-(4-butylpiperazin-1-yl)benzonitrile: ${ }^{21}$ The title compound was preapred according using method A, $74 \%$ yield. ${ }^{1} H$ NMR (400 MHz, MeOD-d4) $\delta 7.58(d, J=8.96 \mathrm{~Hz}, 2 \mathrm{H}), 7.08(\mathrm{~d}, \mathrm{~J}=8.88 \mathrm{~Hz}, 2 \mathrm{H}), 3.55$ (bs, 4H), 3.12 (broad s, $4 \mathrm{H}), 2.89$ (bs, 2H), 1.70-1.68 ( $m, 2 H)$, 1.49-1.40 (sex, $2 H), 1.02(t, J=7.36 \mathrm{~Hz}, 3 H)$; $M S\left(L C / M S, M+H^{+}\right): 245$, HPLC purity: 98\%.

Example 5o: 1-butyl-4-(2-chlorophenyl)piperazine: The title compound was preapred according using method A, 67\% yield. ${ }^{1} H$ NMR (400 $\left.M H z, C D C l_{3}\right) \delta 7.22(d d, J=6.36 \mathrm{~Hz}, 1.52,1 \mathrm{H}), 7.07(t d, J=7.56 \mathrm{~Hz}, 1.56,1 \mathrm{H}), 6.92(d d, J=6.56,1.48 \mathrm{~Hz}, 1 \mathrm{H}), 6.82(t d, J=6.08,1.52$ $\mathrm{Hz}, 1 \mathrm{H}), 2.96(\mathrm{bs}, 4 \mathrm{H}), 2.51(\mathrm{bs}, 4 \mathrm{H}), 2.28(\mathrm{t}, \mathrm{J}=7.64 \mathrm{~Hz}, 2 \mathrm{H}), 1.43-1.35(\mathrm{q}, 2 \mathrm{H}), 1.27-1.18(\mathrm{~m}, 2 \mathrm{H}), 0.83-0.79(t, J=7.32 \mathrm{~Hz}, 3 \mathrm{H}) ; \mathrm{MS}$ $\left(L C / M S, M+H^{+}\right): 254$, HPLC purity: $98 \%$.

Example 6o: 1-butyl-4-(3-chlorophenyl)piperazine: ${ }^{22}$ The title compound was preapred according using method A, $77 \%$ yield. ${ }^{1} H N M R$ (400 MHz, MeOD-d4) $\delta 7.25(q, J=8 \mathrm{~Hz}, 1 \mathrm{H}), 7.02(\mathrm{~s}, 1 \mathrm{H}), 6.93(\mathrm{~d}, \mathrm{~J}=7.92 \mathrm{~Hz}, 1 \mathrm{H}), 6.89(\mathrm{~d}, \mathrm{~J}=7.64 \mathrm{~Hz}, 1 \mathrm{H}), 3.45$ (bs, $4 \mathrm{H}), 3.29(\mathrm{bs}$, 4H), 3.05 (bs, 2H), 1.74 (bs, $2 H), 1.44(b s, 2 H), 1.01(t, J=6.88 \mathrm{~Hz}, 3 H)$; $M S$ (LC/MS, $\left.M+H^{+}\right): 253$, HPLC purity: 98\%.

Example 7o: 1-butyl-4-(4-chlorophenyl)piperazine: ${ }^{23}$ The title compound was preapred according using method A, $69 \%$ yield. ${ }^{1} H N M R$ $\left(400 \mathrm{MHz}, \mathrm{CDCl}_{3}\right) \delta 7.22(\mathrm{~d}, J=8.92 \mathrm{~Hz}, 2 \mathrm{H}), 6.84(\mathrm{~d}, \mathrm{~J}=8.92 \mathrm{~Hz}, 2 \mathrm{H}), 3.23(t, J=4.72 \mathrm{~Hz}, 4 \mathrm{H}), 2.71(t, J=4.88 \mathrm{~Hz}, 4 \mathrm{H}), 2.49(t, J=$ $7.72 \mathrm{~Hz}, 2 \mathrm{H}), 1.57(\mathrm{~m}, J=15.2,8 \mathrm{~Hz}, 2 \mathrm{H}), 1.37(\mathrm{~m}, J=18.4,7.44 \mathrm{~Hz}, 7.40,2 \mathrm{H}), 0.96(t, J=7.32 \mathrm{~Hz}, 3 \mathrm{H}) ; \mathrm{MS}\left(L C / M S, M+H^{+}\right): 254$, HPLC purity: $98 \%$.

Example 9o: 1-butyl-4-(2,4-dichlorophenyl)piperazine: The title compound was preapred according using method A, $71 \%$ yield. ${ }^{1} H$ NMR $\left(400 \mathrm{MHz}, C D C l_{3}\right) \delta 7.35(d, J=2.4 \mathrm{~Hz}, 1 \mathrm{H}), 7.17(d d, J=6.16,2.44 \mathrm{~Hz}, 1 \mathrm{H}), 6.96(d, J=8.64 \mathrm{~Hz}, 1 \mathrm{H}), 3.05(b s, 4 \mathrm{H}), 2.63(b s, 4 H)$, $2.41(t, J=9.84 \mathrm{~Hz}, 2 H), 1.55-1.31(\mathrm{~m}, 2 H), 1.40-1.31(\mathrm{~m}, 2 H), 0.94(t, J=7.32,3 H) ; M S\left(L C / M S, M+H^{+}\right): 288$, HPLC purity: $98 \%$.

Example 10o: 1-butyl-4-(3,5-dichlorophenyl)piperazine: The title compound was preapred according using method A, $81 \%$ yield. ${ }^{l} H N M R$ $\left(400 \mathrm{MHz}, \mathrm{CDCl}_{3}\right) \delta 8.01(\mathrm{~d}, \mathrm{~J}=3.04 \mathrm{~Hz}, 1 \mathrm{H}), 7.23-7.11(\mathrm{~m}, 1 \mathrm{H}), 6.58-6.55(\mathrm{dd}, \mathrm{J}=5.76,3.32 \mathrm{~Hz}, 1 \mathrm{H}), 3.45(\mathrm{t}, \mathrm{J}=5.08 \mathrm{~Hz}, 3 \mathrm{H}), 3.16(t$, $J=5 \mathrm{~Hz}, 1 \mathrm{H}), 2.56-2.32(\mathrm{~m}, 4 H), 2.34(\mathrm{t}, J=7.6 \mathrm{~Hz}, 2 \mathrm{H}), 1.52-1.44(\mathrm{~m}, 2 \mathrm{H}), 1.36-1.27(\mathrm{~m}, 2 \mathrm{H}), 0.90(\mathrm{t}, J=7.32,3 H) ; M S(L C / M S$, $\left.M+H^{+}\right): 288$, HPLC purity: $98 \%$.

Example 11o: 1-butyl-4-(2-methoxyphenyl)piperazine: ${ }^{24}$ The title compound was preapred according using method A, $70 \%$ yield. ${ }^{l} H$ NMR $\left(400 \mathrm{MHz}, C D \mathrm{Cl}_{3}\right) \delta$ 7.02-6.90 (m, 3H), $6.87(\mathrm{dd}, \mathrm{J}=6.56,1.32 \mathrm{~Hz}, 1 \mathrm{H}), 3.87(\mathrm{~s}, 3 \mathrm{H}), 3.12(\mathrm{bs}, 4 \mathrm{H}), 2.67(\mathrm{bs}, 4 \mathrm{H}), 2.42(\mathrm{t}, J=6.6$ $H z, 2 H), 1.52-1.49(m, 2 H), 1.41-1.32(m, 2 H), 0.95(t, J=7.28 H z, 3 H)$; $M S\left(L C / M S, M+H^{+}\right): 250$, HPLC purity: $98 \%$.

Example 12o: 1-butyl-4-(3-methoxyphenyl)piperazine: The title compound was preapred according using method A, $73 \%$ yield. ${ }^{l} H N M R$ $\left(400 \mathrm{MHz}, \mathrm{CDCl}_{3}\right) \delta 7.20(\mathrm{t}, \mathrm{J}=8.12 \mathrm{~Hz}, 1 \mathrm{H}), 6.51(\mathrm{t}, \mathrm{J}=9.04 \mathrm{~Hz}, 2 \mathrm{H}), 6.46(\mathrm{~s}, 1 \mathrm{H}), 3.80(\mathrm{bs}, 3 \mathrm{H}), 3.46(\mathrm{bs}, 4 \mathrm{H}), 3.21(\mathrm{bs}, 4 \mathrm{H}), 2.93(\mathrm{bs}$, 2H), 1.74 (bs, 2H), 1.39 (bs, 2H), $0.97(t, J=7.04 \mathrm{~Hz}, 3 H)$; $M S$ (LC/MS, $\left.M+H^{+}\right): 250$, HPLC purity: 98\%.

Example 13o: 1-butyl-4-(4-methoxyphenyl)piperazine: ${ }^{25}$ The title compound was preapred according using method A, 78\% yield. 1-butyl4-(4-methoxyphenyl) piperazine: ${ }^{1} \mathrm{H} \mathrm{NMR}\left(400 \mathrm{MHz}, \mathrm{CDCl}_{3}\right) \delta 6.99(d, J=8.84 \mathrm{~Hz}, 2 \mathrm{H}), 6.88(d, J=8.84 \mathrm{~Hz}, 2 \mathrm{H}), 3.76(\mathrm{~s}, 3 \mathrm{H}), 3.37-$ $3.32(\mathrm{~m}, 8 H), 3.12(\mathrm{bs}, 2 H), 1.77(\mathrm{bs}, 2 H), 1.48-1.43(\mathrm{~m}, 2 H), 1.03(\mathrm{t}, J=7.16 \mathrm{~Hz}, 3 H)$; $M S\left(L C / M S, M+H^{+}\right): 250$, HPLC purity: 98\%. 
Example 140: 2-(4-butylpiperazin-1-yl)phenol: The title compound was preapred according using method B, $65 \%$ yield. ${ }^{1} H$ NMR (400 $M H z, M e O D-d 4) \delta 7.04(d, J=7.84 \mathrm{~Hz}, 1 \mathrm{H}), 7.00(t, J=14.16 \mathrm{~Hz}, 1 \mathrm{H}), 6.85(d, J=7.64 \mathrm{~Hz}, 2 \mathrm{H}), 3.49$ (broad s, 4H), 3.34-3.30 (m, 4H), $3.22(t, J=7.88 \mathrm{~Hz}, 2 H), 1.84-1.76(\mathrm{~m}, 2 \mathrm{H}), 1.53-1.43(\mathrm{~m}, 2 H), 1.04(t, J=7.28 \mathrm{~Hz}, 3 H)$; $M S\left(L C / M S, M+H^{+}\right): 235$, HPLC purity: 98\%.

Example 15o: 3-(4-butylpiperazin-1-yl)phenol: The title compound was preapred according using method B, 63\% yield. ${ }^{1} H$ NMR (400 $\left.M H z, C D C l_{3}\right) \delta 7.06(t, J=8.04 \mathrm{~Hz}, 1 \mathrm{H}), 6.44(d d, J=6.64,1.2 \mathrm{~Hz}, 1 \mathrm{H}), 6.32(d, J=2.2 \mathrm{~Hz}, 1 \mathrm{H}), 6.29(d, J=1.72 \mathrm{~Hz}, 1 \mathrm{H}), 3.16(t, J=$ $4.32 \mathrm{~Hz}, 4 H), 2.65(t, J=4.56 \mathrm{~Hz}, 4 H), 2.43(t, J=10.56 \mathrm{~Hz}, 2 \mathrm{H}), 1.59-1.52(\mathrm{~m}, 2 \mathrm{H}), 1.38-1.29(\mathrm{~m}, 2 \mathrm{H}), 0.93(t, J=7.28 \mathrm{~Hz}, 3 \mathrm{H}) ; \mathrm{MS}$ $\left(L C / M S, M+H^{+}\right): 235$, HPLC purity: $98 \%$.

Example 16o: 4-(4-butylpiperazin-1-yl)phenol: The title compound was preapred according using method B, 71\% yield. ${ }^{1} H N M R(400$ $M H z, M e O D-d 4) \delta 6.80(d d, J=4.44,2.24 \mathrm{~Hz}, 2 \mathrm{H}), 6.64(d d, J=4.4,2.28 \mathrm{~Hz}, 1 \mathrm{H}), 3.21(\mathrm{bs}, 4 \mathrm{H}), 3.18(\mathrm{bs}, 4 \mathrm{H}), 3.07-3.03(\mathrm{~m}, 2 \mathrm{H}), 1.69-$ $1.62(\mathrm{~m}, 2 \mathrm{H}), 1.40-1.31(\mathrm{~m}, 2 \mathrm{H}), 0.92(t, J=7.36 \mathrm{~Hz}, 3 H) ; M S\left(L C / M S, M+H^{+}\right): 235$, HPLC purity: 98\%.

Example 17o: 1-butyl-4-(o-tolyl)piperazine: ${ }^{26}$ The title compound was preapred according using method A, 66\% yield. ${ }^{1} \mathrm{H} \mathrm{NMR}(400 \mathrm{MHz}$, $\left.C D C l_{3}\right) \delta 7.08(d, J=7.36 \mathrm{~Hz}, 1 \mathrm{H}), 7.06(d, J=7.4 \mathrm{~Hz}, 1 \mathrm{H}), 6.95(d, J=7.6 \mathrm{~Hz}, 1 \mathrm{H}), 6.88(t d, J=7.28,0.92 \mathrm{~Hz}, 1 \mathrm{H}), 2.87(t, J=4.72$ $H z, 4 H), 2.52(b s, 4 H), 2.32(t, J=9.88 H z, 2 H), 2.22(s, 3 H), 1.48-1.42(m, 2 H), 1.32-1.22(m, 2 H), 0.86(t, J=7.32 H z, 3 H) ; M S$ $\left(L C / M S, M+H^{+}\right): 234.20$, HPLC purity: $98 \%$.

Example 180: 1-butyl-4-(m-tolyl)piperazine: The title compound was preapred according using method A, 68\% yield. ${ }^{1} \mathrm{H} \mathrm{NMR}(400 \mathrm{MHz}$, $\left.C D C l_{3}\right) \delta 7.19(t, J=7.76 \mathrm{~Hz}, 1 \mathrm{H}), 6.78(d, J=8.48 \mathrm{~Hz}, 2 \mathrm{H}), 6.73(\mathrm{~d}, J=7.48 \mathrm{~Hz}, 1 \mathrm{H}), 3.25(t, J=4.96 \mathrm{~Hz}, 4 \mathrm{H}), 2.64(t, J=5.08 \mathrm{~Hz}$, $4 H), 2.43(t, J=7.6 \mathrm{~Hz}, 2 H), 2.37(\mathrm{~s}, 3 H), 1.61-1.53(\mathrm{~m}, 2 \mathrm{H}), 1.44-1.36(\mathrm{sex}, 2 \mathrm{H}), 1.00(t, J=7.32 \mathrm{~Hz}, 3 \mathrm{H}) ; \mathrm{MS}\left(L C / M S, M+H^{+}\right): 234$, HPLC purity: $98 \%$.

Example 19o: 1-butyl-4-(p-tolyl)piperazine: ${ }^{27}$ The title compound was preapred according using method A, $72 \%$ yield. ${ }^{1} \mathrm{H} \mathrm{NMR}(400 \mathrm{MHz}$, $\left.C D l_{3}\right) \delta 7.10(d, J=8.2 \mathrm{~Hz}, 2 \mathrm{H}), 6.87(d, J=8.56 \mathrm{~Hz}, 2 \mathrm{H}), 3.22(t, J=4.88 \mathrm{~Hz}, 4 \mathrm{H}), 2.68(t, J=4.68 \mathrm{~Hz}, 4 \mathrm{H}), 2.46(t, J=7.68 \mathrm{~Hz}, 2 \mathrm{H})$, $2.29(\mathrm{~s}, 3 H), 1.61-1.54(\mathrm{~m}, 2 H), 1.42-1.33(\mathrm{~m}, 2 H), 0.96(t, J=7.32 \mathrm{~Hz}, 3 H) ; M S\left(L C / M S, M+H^{+}\right): 234$, HPLC purity: $98 \%$.

Example 20o: 1-butyl-4-(2-(trifluoromethyl)phenyl)piperazine: The title compound was preapred according using method A, $62 \%$ yield. ${ }^{l} \mathrm{H} \mathrm{NMR}\left(400 \mathrm{MHz}, \mathrm{CDCl}_{3}\right) \delta 7.60(d d, J=6.68,1.2 \mathrm{~Hz}, 1 \mathrm{H}), 7.49(\mathrm{td}, J=7.16,1 \mathrm{~Hz}, 1 \mathrm{H}), 7.37(d, J=8 \mathrm{~Hz}, 1 \mathrm{H}), 7.19(t, J=7.6 \mathrm{~Hz}$, $1 H)$, ; $M S\left(L C / M S, M+H^{+}\right): 288$, HPLC purity: $98 \%$.

Example 210: 1-butyl-4-(3-(trifluoromethyl)phenyl)piperazine: ${ }^{27}$ The title compound was preapred according using method A, ${ }^{1} H N M R$ $\left(400 \mathrm{MHz}, \mathrm{CDCl}_{3}\right) \delta 7.30(\mathrm{t}, J=7.96 \mathrm{~Hz}, 1 \mathrm{H}), 7.12(\mathrm{~s}, 1 \mathrm{H}), 7.03(\mathrm{td}, J=6.16,2.28 \mathrm{~Hz}, 2 \mathrm{H}), 3.22(\mathrm{t}, J=5 \mathrm{~Hz}, 4 \mathrm{H}), 2.7(t, J=5.12 \mathrm{~Hz}$, $4 H), 2.36(t, J=9.6 \mathrm{~Hz}, 2 \mathrm{H}), 1.55-1.47(\mathrm{~m}, 2 \mathrm{H}), 1.40-1.31(\mathrm{sex}, 2 \mathrm{H}), 0.94(t, J=7.32 \mathrm{~Hz}, 3 \mathrm{H}) ; \mathrm{MS}\left(L C / M S, M+H^{+}\right): 288$, HPLC purity: $98 \%$.

Example 22o: 1-butyl-4-(4-(trifluoromethyl)phenyl)piperazine: The title compound was preapred according using method A, ${ }^{1} H N M R$ $\left(400 \mathrm{MHz}, \mathrm{CDCl}_{3}\right) \delta 7.38(\mathrm{~d}, J=8.68 \mathrm{~Hz}, 2 \mathrm{H}), 6.83(\mathrm{~d}, \mathrm{~J}=8.72 \mathrm{~Hz}, 1 \mathrm{H}), 3.21(\mathrm{t}, J=5.08 \mathrm{~Hz}, 4 \mathrm{H}), 2.51(\mathrm{t}, \mathrm{J}=5.12 \mathrm{~Hz}, 4 \mathrm{H}), 2.33-2.29(\mathrm{t}$, $J=7.56 \mathrm{~Hz}, 2 \mathrm{H}), 1.47-1.40(\mathrm{~m}, 2 \mathrm{H}), 1.32-1.23(\mathrm{~m}, 2 \mathrm{H}), 0.86(\mathrm{t}, J=7.32 \mathrm{~Hz}, 3 \mathrm{H}) ; \mathrm{MS}\left(L C / M S, M+H^{+}\right): 288$, HPLC purity: $98 \%$.

Supporting Table S1. The average fractions of three trajectories of each complex simulation in which the ligands are in contact $(<5 \AA$ distance) specific residues. The errors represent population standard deviations.

\begin{tabular}{ll|cccccc}
\hline System & Experimenta & \multicolumn{5}{|c}{ Average fraction of trajectories in contacts with the ligands } \\
\cline { 3 - 8 } & $\mathbf{I ~ k}_{\mathbf{i}} \mathbf{( n M )}$ & Trp $^{\text {ECL1 }}$ & Cys $^{\text {ECL2 }}$ & Ile $^{\text {ECL2 }}$ & Trp $^{6.48}$ & His $^{6.55}$ & Tyr $^{7.15}$ \\
\hline D2R-10 & $22.3 \pm 4.9$ & $0.001 \pm 0.001$ & $0.000 \pm 0.000$ & $0.000 \pm 0.000$ & $0.000 \pm 0.000$ & $0.004 \pm 0.001$ & $0.007 \pm 0.002$ \\
D2R-10a & $27.7 \pm 5.4$ & $0.014 \pm 0.012$ & $0.000 \pm 0.000$ & $0.000 \pm 0.000$ & $0.000 \pm 0.000$ & $0.109 \pm 0.040$ & $0.036 \pm 0.006$ \\
D2R-10b & $0.26 \pm 0.05$ & $0.069 \pm 0.010$ & $0.001 \pm 0.000$ & $0.023 \pm 0.000$ & $0.000 \pm 0.000$ & $0.054 \pm 0.060$ & $0.089 \pm 0.011$
\end{tabular}




\begin{tabular}{ll|llllll} 
D2R-2o & $150 \pm 56.1$ & $0.001 \pm 0.011$ & $0.000 \pm 0.000$ & $0.000 \pm 0.000$ & $0.003 \pm 0.000$ & $0.013 \pm 0.028$ & $0.005 \pm 0.014$ \\
D2R-2oa & $743 \pm 77$ & $0.060 \pm 0.022$ & $0.000 \pm 0.001$ & $0.000 \pm 0.022$ & $0.000 \pm 0.000$ & $0.030 \pm 0.039$ & $0.148 \pm 0.047$ \\
\hline D3R-1o & $14.7 \pm 1.2$ & $0.003 \pm 0.059$ & $0.001 \pm 0.001$ & $0.019 \pm 0.019$ & $0.004 \pm 0.000$ & $0.004 \pm 0.023$ & $0.021 \pm 0.037$ \\
D3R-1oa & $0.17 \pm 0.01$ & $0.000 \pm 0.056$ & $0.000 \pm 0.000$ & $0.033 \pm 0.017$ & $0.001 \pm 0.000$ & $0.114 \pm 0.043$ & $0.051 \pm 0.055$ \\
D3R-1ob & $13.5 \pm 3.5$ & $0.005 \pm 0.065$ & $0.278 \pm 0.001$ & $0.040 \pm 0.007$ & $0.002 \pm 0.003$ & $0.006 \pm 0.046$ & $0.048 \pm 0.049$ \\
D3R-2o & $30.5 \pm 12.8$ & $0.000 \pm 0.004$ & $0.032 \pm 0.000$ & $0.036 \pm 0.002$ & $0.004 \pm 0.003$ & $0.021 \pm 0.009$ & $0.002 \pm 0.005$ \\
D3R-2oa & $0.5 \pm 1.1$ & $0.000 \pm 0.001$ & $0.020 \pm 0.000$ & $0.008 \pm 0.000$ & $0.002 \pm 0.003$ & $0.119 \pm 0.009$ & $0.127 \pm 0.005$ \\
\hline
\end{tabular}

The Amber input files used for equilibration steps and the production simulations:

\section{Step 1:}

Min explicit solvent heavy atom rest no shake

\&cntrl

$\operatorname{imin}=1, \operatorname{ntmin}=2, \operatorname{maxcyc}=1000$,

ntwx $=500$, ioutfm $=1, n$ tpr $=50$, ntwr $=500$,

ntc $=1, \mathrm{ntf}=1, \mathrm{ntb}=1$, cut $=8.0$,

igb $=0$, saltcon $=0.0$,

ntr = 1, restraintmask = ':1-\$S,672 \&!@H=', restraint_wt = 5.0,

\&end

\section{Step 2:}

MD explicit solvent heavy atom rest shake dt 0.001

\&cntrl

$\operatorname{imin}=0, \mathrm{nstlim}=15000, \mathrm{dt}=0.001$,

$\mathrm{ntx}=1$, irest $=0$, ig $=-1$,

ntwx $=500$, ioutfm $=1$, ntpr $=50$, ntwr $=500$,

iwrap $=1, \mathrm{nscm}=0$,

ntc $=2$, ntf $=1, \mathrm{ntb}=1$, cut $=8.0$,

$\mathrm{ntt}=1$, tautp $=0.5$, temp0 $=\$ \mathrm{~T}$, tempi $=\$ \mathrm{~T}$,

ntp $=0$, taup $=0.5$,

igb $=0$, saltcon $=0.0$,

ntr = 1, restraintmask = ':1-\$S,672 \&!@H=', restraint_wt = 5.0,

\&end

\section{Step 3:}

\&cntrl

$\operatorname{imin}=1, n t \min =2, \operatorname{maxcyc}=1000$,

ntwx $=500$, ioutfm $=1$, ntpr $=50$, ntwr $=500$,

ntc $=1, \mathrm{ntf}=1, \mathrm{ntb}=1$, cut $=8.0$,

igb $=0$, saltcon $=0.0$, 
$\mathrm{ntr}=1$, restraintmask = ':1-\$S,672 \&!@H=', restraint_wt = 2.0,

\&end

\section{Step 4:}

\&cntrl

$\operatorname{imin}=1, n \min =2, \operatorname{maxcyc}=1000$,

ntwx $=500$, ioutfm $=1$, ntpr $=50$, ntwr $=500$,

$\mathrm{ntc}=1$, ntf $=1, \mathrm{ntb}=1$, cut $=8.0$,

igb $=0$, saltcon $=0.0$,

ntr = 1, restraintmask = ':1-\$S,672 \&!@H=', restraint_wt = 0.1,

\&end

\section{Step 5:}

\&cntrl

$\operatorname{imin}=1, n t \min =2, \operatorname{maxcyc}=1000$,

ntwx $=500$, ioutfm $=1$, ntpr $=50$, ntwr $=500$,

ntc $=1$, ntf $=1, \mathrm{ntb}=1$, cut $=8.0$,

igb $=0$, saltcon $=0.0$,

$n t r=0$,

\&end

\section{Step 6:}

\&cntrl

$\operatorname{imin}=0, n s t l i m=5000, d t=0.001$,

ntx $=1$, irest $=0$, ig $=-1$,

ntwx $=500$, ioutfm $=1$, ntpr $=50$, ntwr $=500$,

iwrap $=1, \mathrm{nscm}=0$,

ntc $=2$, ntf $=1$, ntb $=2$, cut $=8.0$,

ntt $=1$, tautp $=1.0$, temp $0=\$ T$, tempi $=\$ T$,

ntp $=1$, taup $=1.0$,

igb $=0$, saltcon $=0.0$,

ntr $=1$, restraintmask = ':1-\$S,672 \& !@H=', restraint_wt = 1.0,

\section{\&end}

\section{Step 7:}

\&cntrl

$\operatorname{imin}=0, n s t l i m=5000, d t=0.001$,

$n t x=5$, irest $=1$,

ntwx $=1000$, ioutfm $=1$, ntpr $=1000$, ntwr $=1000$,

iwrap $=1, \mathrm{nscm}=0$,

ntc $=2$, ntf $=1$, ntb $=2$, cut $=8.0$,

$\mathrm{ntt}=1$, tautp $=1.0$, temp $0=\$ \mathrm{~T}$, tempi $=\$ \mathrm{~T}$,

ntp $=1$, taup $=1.0$,

igb $=0$, saltcon $=0.0$, 
ntr $=1$, restraintmask = ':1-\$S,672 \&!@H=', restraint_wt = 0.5,

\&end

\section{Step 8:}

\&cntrl

$\operatorname{imin}=0, n s t l i m=10000, d t=0.001$,

$n t x=5$, irest $=1$,

ntwx $=1000$, ioutfm =1, ntpr = 1000, ntwr = 1000,

iwrap $=1, \mathrm{nscm}=0$,

ntc $=2$, ntf $=1, \mathrm{ntb}=2$, cut $=8.0$,

ntt $=1$, tautp $=1.0$, temp $0=\$ T$, tempi $=\$ T$,

ntp $=1$, taup $=1.0$,

igb $=0$, saltcon $=0.0$,

ntr $=1$, restraintmask = "\$BACKBONEMASK", restraint_wt $=0.5$,

\&end

\section{Step 9:}

\&cntrl

$\operatorname{imin}=0$, nstlim $=500000, \mathrm{dt}=0.002$,

$\mathrm{ntx}=5$, irest $=1$,

ntwx $=5000$, ioutfm $=1$, ntpr $=5000$, ntwr $=5000$,

iwrap $=1, \mathrm{nscm}=1000$,

ntc $=2$, ntf $=1, \mathrm{ntb}=2$, cut $=8.0$,

$\mathrm{ntt}=1$, tautp $=1.0$, temp0 $=\$ \mathrm{~T}$, tempi $=\$ \mathrm{~T}$,

ntp $=1$, taup $=1.0$,

igb $=0$, saltcon $=0.0$,

ntr $=0$, nmropt $=1$,

\&end

\&wt

type='END',

\&end

DISANG=salt_bridge.disang

The mdin file for a 300ns chunk of the production:

\&cntrl

$\operatorname{imin}=0, n s t l i m=75000000, d t=0.004$,

irest $=1, \mathrm{ntx}=5$, ig $=-1$,

ntwx $=2500$, ioutfm $=1$, ntpr $=2500$, ntwr $=2500$, ntxo $=2$,

iwrap $=1, \mathrm{nscm}=1000$, tol=0.0000001, ntc $=2, \mathrm{ntf}=2$, ntb $=2$, cut = 9.0,

$\mathrm{ntt}=3$, gamma_In $=1$, temp $0=310.15$, tempi $=310.15$,

ntp $=1$, barostat $=2$, taup $=5.0$,

nmropt $=1$,

\&end

\&wt 
type='END',

\&end

DISANG=salt_bridge.disang

The salt bridge restraint file (salt_bridge.disang):

\&rst

iat=1229,19782, r1=0.000, r2=02.800, r3=05.800, r4=999.000, rk2=10.0, rk3=10.0,

\&end

The cpptraj script used for calculating the RDF plots:

parm ../build/full.topo [traj]

trajin ../prod/traj.*

reference ../build/full.pdb parm [traj] [xtal]

autoimage origin

rmsd rmsRec ref [xtal] :1-185,194-272\&!@H=

radial rdf_wat_pocket.dat 0.2520 :WAT@O :47-52@CA,79-84@CA,162-166@CA,244-249@CA center2 volume \# For D3R systems

radial rdf_wat_pocket.dat 0.2520 :WAT@o :49-54@CA,80-85@CA,160-164@CA,240-245@CA center2 volume \# For D2R systems 